\title{
Design and Verification for Hypersonic Aerodynamic Model with Duct of Internal and External Flow Decoupling
}

\author{
Zejiang Wang ${ }^{1,2}$, Wenping Song ${ }^{1}$, Jin Jiang ${ }^{2}$, Huiyong Zhao ${ }^{2} \&$ Yong Zhang ${ }^{2}$ \\ ${ }^{1}$ School of Aeronautics, Northwestern Polytechnical University, Xi'an, China \\ ${ }^{2}$ Science and Technology on Scramjet Laboratory, Hypervelocity Aerodynamics Institute of CARDC, Sichuan, \\ Mianyang, China \\ Correspondence: Jin Jiang, Science and Technology on Scramjet Laboratory, No. 278, Jianmen Road, Mianyang, \\ China. E-mail: jijnpu@163.com
}

Received: May 16, 2015

Accepted: July 15, 2015

Online Published: October 31, 2015

doi:10.5539/mas.v9n12p202

URL: http://dx.doi.org/10.5539/mas.v9n12p202

\begin{abstract}
In order to develop the design techniques for the hypersonic aerodynamic testing model with duct system of internal flow and external flow decoupling, this research used a circular cross section air-breathing hypersonic cruise vehicle model, explored the model design technique for internal flow and external flow separated from each other, the design technique and the seal technique for the clearance between the internal flow part and the external flow part, the design technique of ring type six-component strain-gauge balance and so on. A wind tunnel test was conducted at mach 6 . The results of the test indicate that, the design of the internal flow and external flow decoupling test system is successful to get credible test data. It have been mastered that the key techniques for the test system design of internal flow and external flow decoupling.
\end{abstract}

Keywords: hypersonic, wind tunnel tests, circular cross section scramjet, model design, verification

\section{Introduction}

For scramjet-powered air-breathing hypersonic vehicle, internal flow and external flow are highly coupled(Holland,Woods\& Engelund,2001;Le,2010), the cold internal flow not only is closely related to engine performance, but also is closely associated with the aerodynamic performance(Le,2010; Luo,Xu,\& Liu,2007; Bai\&Wang,2012;Le,2010;Luo,Huang,Jing,\&Wang,2010). Both aerodynamic performance prediction(Mitani et al.,2001; Wang,2004; Anderson,1983; Pinckney,1978) and engine thrust assessments(Chen\&Xia,2004; Engelund,Holland,\&Charles,2000;Voland,1990) are required to obtain high-precision internal and external flow aerodynamic ground test data. In ground tests, it is critical access for internal and external flow aerodynamic balance measurement data to separate the highly coupled internal flow from external flow and decouple the internal and external aerodynamic force.

So far, the internal and external flow decoupling technique has not yet been solved well, and the test data of the integrated aerodynamic/propulsion performance prediction were met by using a separate internal and external flow aerodynamic load measurement method. Conventional ventilation model dynamometer test, can achieve both full- scale aerodynamic measurement data and internal resistance data based on momentum control volume method, with subtracting between the whole scale data and internal resistance data, can obtain high-precision external flow aerodynamic data, the available data accuracy can fully meet the needs of project development. In the eighties of last century, model design did not solve the problem of internal and external flow coupling, based on the full scale balance measurement data, the wall pressure measurement data and the nozzle outlet parameter measurement data, Anderson (Anderson,1983) and Pinckney (Pinckney,1978) proposed three cold internal flow resistance prediction methods that can accurately assess the cold internal flow aerodynamic data. The previous experimental studies failed to solve the internal and external flow coupling problems so that the internal and external flow aerodynamic loads can't be separated, and the internal and external flow aerodynamic balance measurement can not be obtained directly.

In order to develop the internal flow aerodynamic balance direct measurement test technology, Professor $\mathrm{Xu}(\mathrm{Xu}$, Shu, Xie, \& Zhang, 2013) studied the independent internal flow channel model design of the single-module rectangular section scramjet, mastered the internal and external flow isolation technology that can accurately 
simulate the flow field, got the internal flow aerodynamic three-component balance measurements with accuracy up to $6 \sim 8 \%$. Although this test only solved part of the problem of internal and external flow decoupling technique without the validation test decupling between internal and external flow, it established the foundation for the hypersonic internal and external flow decoupling test system design. In Xu's experiments, with respect to the internal and external flow completely decoupling, since the external flow simulation is not required, the external flow part design is simple; because the internal and external flow component design gap, mutual positioning, balance layout, channeling and other issues are not considered, the test system, balance design and correction technique are relatively simple. In addition, because the rectangular cross-section scramjet engine is completely different from a circular cross-section scramjet engine in configurations, the problem required to solve is not the same for the scramjet engine with circular cross-section to achieve internal and external flow decoupling.

Therefore, this paper is focused on the study of the air-breathing hypersonic cruise vehicle with circular cross-section configuration similar to the Hyfly, the internal and external flow decoupling test system design technology has been studied by using the CFD-aided design methods, including the internal and external flow component design, the internal and external flow component separation design, the gap sealing measures of the internal and external flow parts, and the verification tests conducted at corresponding to $\mathrm{Ma}=6$ flight conditions , the reliability analysis was comparatively conducted. After the technology is mature, the internal and external flow decoupling can be realized, which make it possible that direct measurement of the highly coupled internal and external flow balance aerodynamic load, this can be applied to various types of dynamometer test for the air-breathing hypersonic vehicle, and provide reliable data for the integrated aerodynamic/propulsion performance prediction, which has high-value engineering applications.

\section{Test System Design}

\subsection{Design Requirements and Simulating Criteria}

\subsubsection{Design Requirements}

The test system design was carried out according to the wind tunnel model design requirements of FD-20A ( $\Phi 1 \mathrm{~m}$ hypersonic wind tunnel) in Hypervelocity Aerodynamics Institute of China Aerodynamics Research and Development Center (CARDC).

The test system was abdominally braced to achieve internal and external flow decoupling, which to ensure measuring the internal and external flow aerodynamic loads.

\subsubsection{Similarity Criterion}

Geometric shape similarity: the internal and external flow control interface is divided, and the internal and external flow profile was simulated according to the theory dimensions, to ensure the internal and external flow parts geometrically similar.

Internal and external flow simulation: the inlet flow field was consistent with the real situation, and the inlet flow coefficient was consistent with the real situation as possible.

The plume flow and jet simulation: the plume flow and jet simulation was satisfied by the similar shape and inflow.

Include in these subsections the information essential to comprehend and replicate the study. Insufficient detail leaves the reader with questions; too much detail burdens the reader with irrelevant information. Consider using appendices and/or a supplemental website for more detailed information.

\subsection{Design Ideas and the Overall Program}

Appropriate identification of research participants is critical to the science and practice of psychology, particularly for generalizing the findings, making comparisons across replications, and using the evidence in research syntheses and secondary data analyses. If humans participated in the study, report the eligibility and exclusion criteria, including any restrictions based on demographic characteristics.

(1) Internal and external flow aerodynamic separation: To measure directly the internal and external flow aerodynamic loads, the highly coupled internal and external flow will be physically separated. Therefore, the internal and external flow component parts must be designed according to the internal and external flow control interface based on the force calculation method. The internal and external flows are separated and decoupled physically.

(2) Internal and external flow field simulation: The principle of geometric similarity must be observed, the 
internal and external flow surfaces are simulated by the internal and external flow components in order to obtain the internal and external flow field and get reliable internal and external aerodynamic loads.

(3) Design of gap between components: In order to achieve the internal and external flow decoupling, there must exist gap between the internal and external flow components. The difficulty of parts gap design lies in: where to open gap for the internal and external flow components with minimal impact on the accuracy of the internal and external flow aerodynamic loads; what's the gap size to ensure no force transfer between the internal and external flow components by contact during the test. Therefore, we must adopt the CFD aided design approach to evaluate the gap size and position, and the design was verified and confirmed by experiment.

(4) Part gap seal: In order to achieve the internal and external flow decoupling, the gap between the components must be sealed to avoid channeling impact on the measurement results. Meanwhile the sealing method should be considered the interference of sealing in balance force measurement. Seal without passing force and interfering can ensure the accuracy of the balance measurement data. Therefore, it's necessary to carefully consider the gap sealing method with enough techniques to monitor, verify and validate its functions.

In summary, the following should be noted for the internal and external decoupling test system design: the CFD-aided design method should be adopted to complete the internal and external component gap design, to ensure the reliability of the internal and external flow decoupling and the accuracy of the internal and external aerodynamic flow measurement; the part head gap can't be located on the opening compression surface of inlet, otherwise the external flow field will be undermined; the parts gap must be sealed by the flexible material to ensure parts not contacting with each other, not channeling the internal and external flow; the pressure monitoring points should be arranged in the part gap cavity to confirm whether the internal and external flow channeling.

The scaled test model has small size but a lot of functions to achieve. It was not only to increase the difficulty of the design and layout for the internal and external flow aerodynamic balance measurement, but also to carefully consider the overall layout, structure, layout and size of the abdominal bracing test system. In order to reduce the weight, the parts wall thickness was as thin as possible.

\subsection{Scale Model Design}

The test model was a circular cross-section configuration of the air-breathing hypersonic cruise vehicle similar to that of Hyfly. According to the FD-20A wind tunnel model design constraints in CARDC, the reduced scale model has the largest diameter of cross-section section to $100 \mathrm{~mm}$ and the nominal length of $850 \mathrm{~mm}$.

\subsubsection{Internal and External Flow Component Design}

In order to achieve the decoupling between internal and external flow, the reduced scale model was divided into the internal and external flow components according to the control interface between internal and external flow as shown in Figure1, and the opening were at the front lip of the engine inlet and the bottom edges. The internal flow components included the inner/outer compression conical surface of inlet, the inlet inner compression cone surface, isolator, combustor and the inner surface of nozzle. The rest surfaces except for the internal flow surfaces belonged to external flow components. The model design of the external flow components had been simplified without rudder, wing, tail fin and other wings. The internal and external flow profile design strictly observed the principle of the geometric similarity.

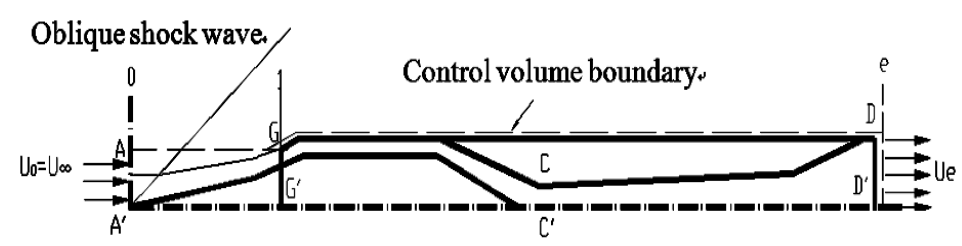

Figure 1. Control volume of circular cross-section scramjet

\subsubsection{The Separation Design of the Internal and External Flow Components}

The internal and external flow components clearance was evaluated by the CFD-aided design tools. On the basis of the assessment results, a variety of factors were weighed, and the components gap opening position and the gap size were reasonably determined, to ensure the internal and external flow decoupled physically. The typical calculated Mach contours were shown in Figure 2. 
The head gap can be opened on the top or bottom wall surface of the lip. The evaluation result showed that the wave structure of the outer hood changed when the opening was located on the wall surface of the lip under the same loss area (Figure 2 (a)), which would have large effect on the test results. When the opening is located on the upper wall surface of the lip, the pneumatic wall would be formed (Figure 2 (b)), which had little effect on the flow structure. With reducing the distance from the head gap to the lip, the loss area of control volume for internal flow was smaller and the measurement accuracy of aerodynamic force was higher. The head gap cannot be too close to the lip edge of inlet, otherwise it would affect the structural strength of the model. Therefore, the best place to the head gap was in the vicinity of the lip of the internal flow channel, which was close to the lip as possible as it can.

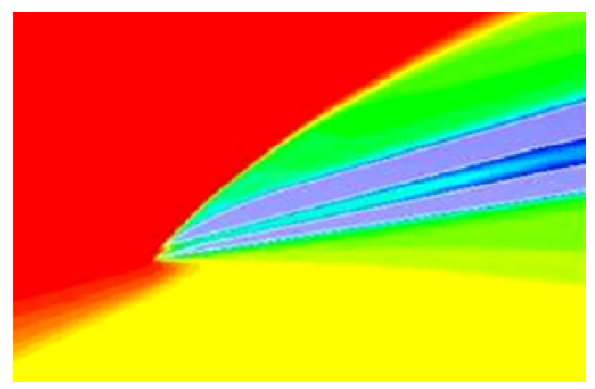

(a) Clearance at the upper wall of lip

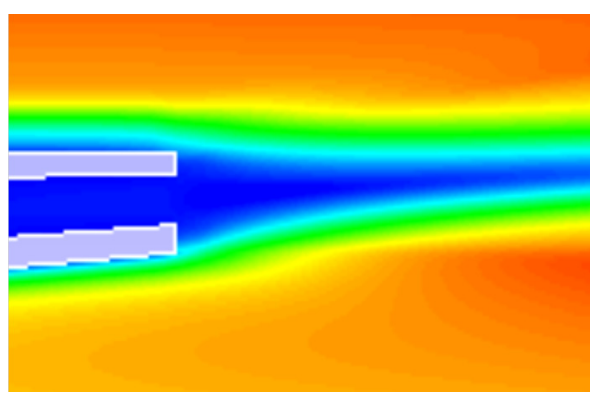

(c) Clearance at the model base

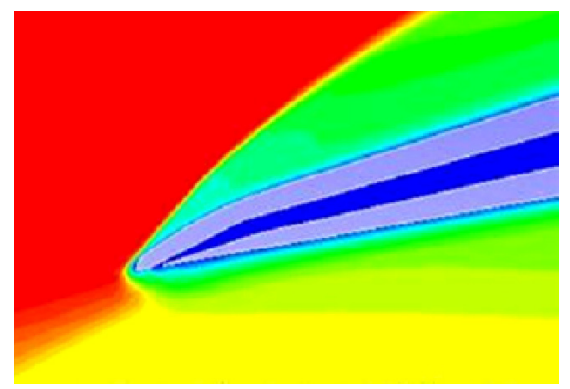

(b) Clearance at the lower wall of lip

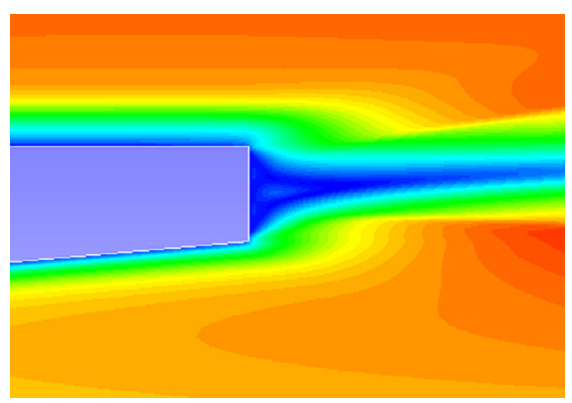

(d) No clearance at the model base

Figure 2. Partial enlarged images of mach number distribution

Based on the assessment result, the head gap size should be moderate. The large head gap would not only affect the structure of the flow field, but also would bring seal difficulty. On the contrary, the smaller gap size would lead to smaller effect on the flow filed, higher aerodynamic measurement accuracy and the better decoupling between the internal and external flow. However, if the gap size was too small, both the internal and external flow components may contact locally during the model installation and test, which resulted in the inaccuracy of the aerodynamic measurements.

With comprehensive consideration of the control volume interface, the analysis result, the structural strength and the size requirement, the head gap was $5 \mathrm{~mm}$ to the inlet lip, the gap size was $2.5 \mathrm{~mm}$, and the head gap width was $2 \mathrm{~mm}$ (Figure3 (a)). The tail gap design was relatively simple. In order to ensure the internal and external flow components not to contact with each other, the tail gap size was $4.9 \mathrm{~mm}$ (Figure 3 (b)). 


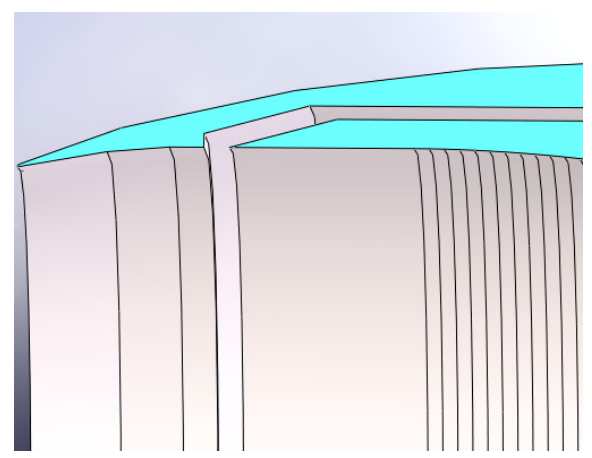

(a) Sketch of the nose clearance

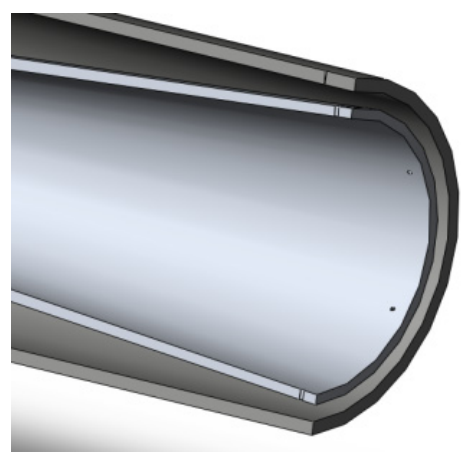

(b) Sketch of the tail clearance

Figure 3. Partial enlarged drawing of the clearance between internal flow parts and external flow parts

\subsubsection{The Gap Sealing Measurement}

By CFD assessment, because the pneumatic wall was formed and the structure of the flow field was not affected, the head gap will not be sealed. The flow structure for the bottom gap with opening (Figure 2 (c)) and without opening (Figure 2 (d)) is different, therefore the bottom gap must be sealed. The soft sealing method was used to meet the requirement of no passing force and small interference. Due to the simple structure of the gap opening, the high temperature silicone rubber can be used for sealing.

\subsubsection{Seal Monitoring}

To confirm the effect of model gap sealing, the pressure measuring points were arranged in the head, middle and tail section of the model gap cavity. The pressure monitoring data can reveal the pressure variation of the gap, which can be used to judge channeling. According to the aerodynamic measurement data of the internal and external flow components, it can be determined whether or not the internal and external flow is channeling.

\subsubsection{Structural Design}

In order to facilitate processing and installation, the modular-assembled design method was used for each component. Among them, the external flow component included the hood section, the isolator housing segment, the outflow balance installation section and the tail section. The internal flow component included the nose cone section, the isolator, the internal flow balance installation segment and the nozzle. The different segments of the various components were connected by the screw threads, and the specific structure was shown in Figure 4.

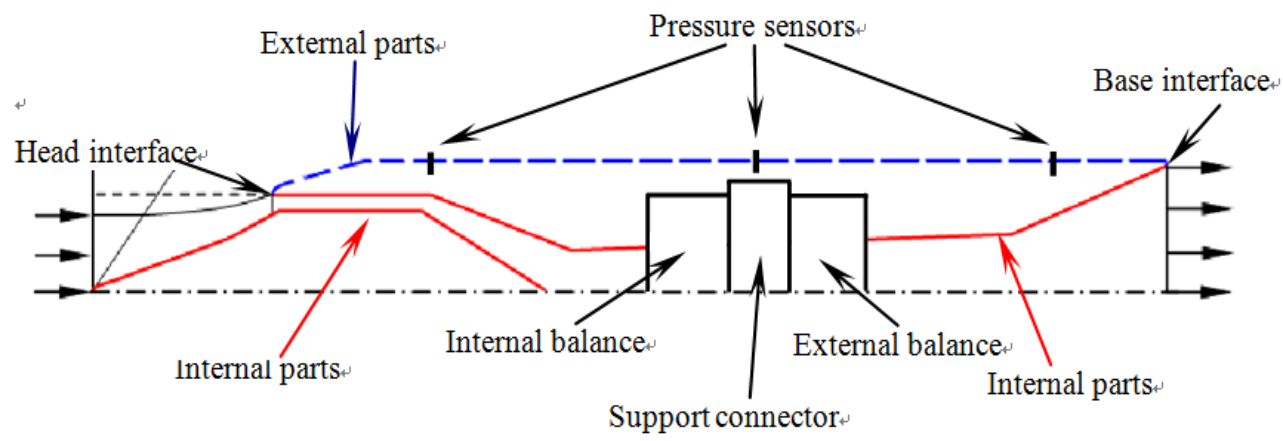

Figure 4. Sketch of the test model

\subsection{Balance Design}

Due to the requirements limit of simultaneously measuring the shape and aerodynamic, the ring structure and in-the-belly support programs were used for the internal and external flow balance (Figure 5), which was installed in the internal and external flow components gap cavity. 


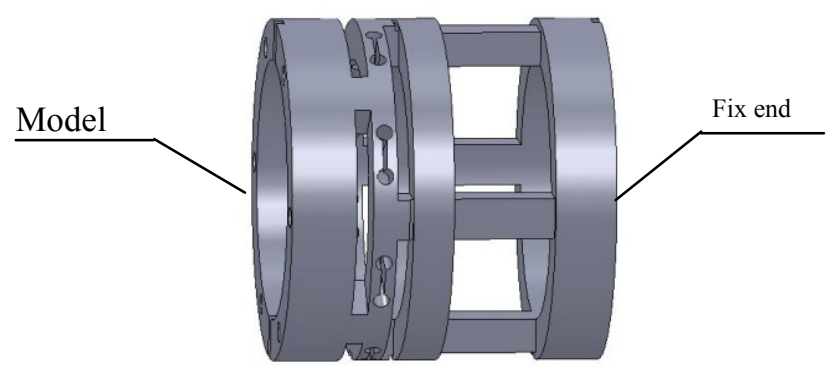

Figure 5. Structure of the ring type strain-gauge balance

The impact load must be considered when the wind tunnel started and closed, and the slender model deformation and other factors must also be considered. The sensitivity of the balance must be increased to improve the accuracy of wind tunnel test dynamometer. The balance dimensions were: axial length of $80 \mathrm{~mm}$, an outer diameter of $\Phi 84 \mathrm{~mm}$, an inner diameter of $\Phi 64 \mathrm{~mm}$. The range and accuracy of six-component ring balance were shown in Table 1.

To avoid the internal and external flow components contact and power transmission with a large scale deformation, the balance design needed a large stiffness and relatively low sensitivity. The structural strength of the balance was checked by the finite element analysis method. The maximum stress is about $4.64 \times 10^{7} \mathrm{~Pa}$, which met the design requirement.

Table 1. Technical index of the ring type strain-gauge balance

\begin{tabular}{ccccccc}
\hline Aerodynamic components & $A / \mathrm{N}$ & $N / \mathrm{N}$ & $Z / \mathrm{N}$ & $M z / \mathrm{N} . \mathrm{m}$ & $M y / \mathrm{N} . \mathrm{m}$ & $M x / \mathrm{N} . \mathrm{m}$ \\
\hline External flow balance & 200 & 400 & 240 & 80 & 48 & 2 \\
Internal flow balance & 100 & 200 & 200 & 10 & 10 & 3 \\
Accuracy & $0.6 \%$ & $0.6 \%$ & $0.6 \%$ & $0.6 \%$ & $0.6 \%$ & $0.7 \%$ \\
\hline
\end{tabular}

Note. $A=$ axial force, $N=$ normal force, $\mathrm{Z}=$ side force, $M z=$ pitching moment, $M y=$ yawing moment, $M x=$ rolling moment.

\subsection{Test System}

The abdominal support mode was adopted in the test system. The model annular bracket fixedly connected with the abdominal support was located at the geometric center of the scaled model, where the front end face was fixed with the internal flow balance and the rear end surface was fixed with the outflow balance.

Because the external flow parts had large aerodynamic force with the action point near the tail section, the external flow balance was rear-installed. Because the internal flow parts had large axial drag and small pitching moment, the internal flow balance was fore-installed. The fixed end and the support ring were fastened, and the floating end was connected to the external and internal components separately by the axial bolts.

\section{Measurement Accuracy Assessments}

For the hypersonic internal and external flow decoupling test system, the factors which may affect measurement accuracy were: the measurement precision of the internal and external flow balance, the impact on the measurement accuracy of the external flow aerodynamic by the abdominal support and the interference of the gap sealing medium.

\subsection{The Abdominal Support Interference Analysis}

The abdominal support didn't interfere with the internal flow, but interfered with the external flow after the bracket(Tang,2004;Yun,1991;Wang,2001), so the external flow measurements must be corrected. The abdominal bracing correction technology was mature with test and CFD methods. In this paper, The CFD simulations were simulated for the test conditions with and without the bracket. The difference between forces with and without the bracket was no more than $3 \%$, which was regarded as interference. After correction, abdominal support external flow aerodynamic interference uncertainty can be controlled at about $1 \%$. There was not the external 
and internal flow channeling for the abdominal support. Without the channeling interference, the measurement accuracy was relatively high.

\subsection{Sealing Medium Interference Analysis}

Since the seal media must contact with the model, it would cause the interference effects on the balance force measurement, so that the effect must be evaluated. The interference on the balance force measurements for the sealing medium mainly had two parts:

\subsubsection{The Interference on the Balance Force Measurements for the Sealing Medium}

The sealing medium had a certain modulus of elasticity, which hindered the action of the strain deformation of balance so as to produce the interference force. For this case, the maximum deformation of the balance may occur during the measurement process. The $\mathrm{X}$ displacement $\Delta \mathrm{L}$ of balance could be obtained under the maximum load with finite element computation. The experimental sealing medium is silica gel $(\mathrm{E}=6 \mathrm{MPa}$, Poisson's ratio of 0.5), combined with a sealing structure specific dimensions, the interference forces $\mathrm{F}$ can be calculated. Namely:

$$
F=E \frac{\Delta L}{L} \cdot S
$$

Where $F$ is the sealing medium interference force (N), $E$ refers to the sealing medium elastic modulus (pa), $S$ refers to the sealing contact area $\left(\mathrm{m}^{2}\right)$, and $L$ is the sealing gap size $(\mathrm{m})$.

The sealing medium force diagram was shown in Figure 6 . The results showed that, due to the larger stiffness of balance, the amount of sealing medium interference was a small magnitude less than $1 \%$ in the case of full-scale strain. Therefore, it can be considered as the amount of system interference without modification.

\subsubsection{The Compressive Deformation Interference of Sealing Medium}

The distortion would be produced for the sealing medium under the flow field and the gap cavity pressure, and the model may be squeezed, and affect the balance force measurement accuracy. Since only two directions were constrained for the gap sealing medium as shown in Figure 6, the seal was deformed under the pressure and would move along the unconstrained direction, the model would not be squeezed, and the interference is negligible.

The average static pressure of the flow field from CFD results in contact with the sealing medium was the same order of magnitude as that of the gap cavity, which was about $10 \mathrm{kPa}$. The pressure difference of the sealing medium was small on both unconstrained direction so as to prevent the sealing medium from falling off during the test, which was the theoretical basis for a good seal.

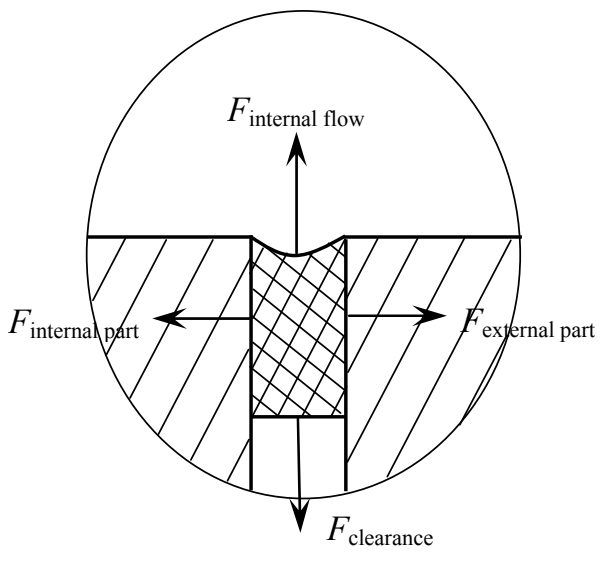

(a) Sketch of the nose clearance

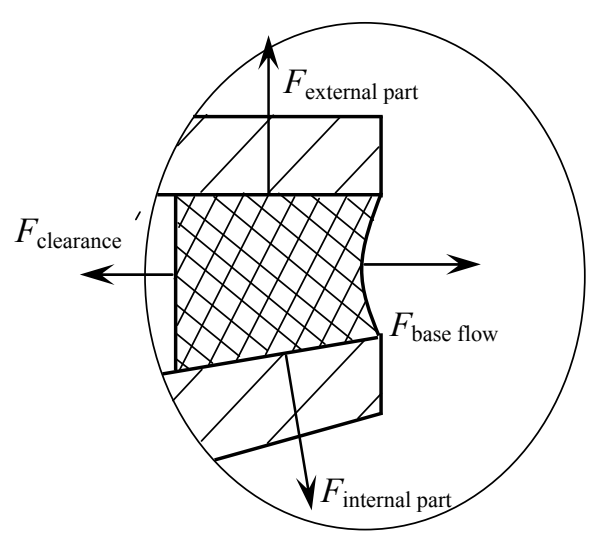

(b) Sketch of the tail clearance

Figure 6. Force diagram of the sealing medium in model clearance

The accuracy of the balance was $0.6 \%$, and the measurement error was less than $1 \%$, which was a small quantity, and it will not be discussed. 
From the above analysis, the dynamometer test measurements and other random error were supposed to be about $3 \%$, so that the measurement error of the internal and external flow aerodynamic were about $3 \%$ and $4 \%$, respectively.

\section{Test Results and Analysis}

To confirm the internal and external flow decoupling effect of the test system design, the verification test was conducted in the FD-20A wind tunnel ${ }^{[14]}$. The experimental conditions included $M a=6.0$, total pressure $P_{0}=$ $2.92 \times 10^{6} \mathrm{pa}$, the angle of attack $\alpha=-6^{\circ} \sim 8^{\circ}$, side slip angle $\beta=0^{\circ}$. With conventional aerodynamic test data processing methods, the aerodynamic coefficients were obtained.

\subsection{Gap seal Verification}

When the gap opening was sealed/un-sealed, the static pressure monitoring curves of three sections in the gap cavity were shown in Figure 7.

From Figure 7, when the gap opening was not sealed, the monitored pressure curve of the three sections (front, middle and rear) was obviously not coincided, where there was spillage flow. When the gap opening was sealed, three monitoring pressure curves were overlapped, and they were sensitive to change in angle of attack, which was proof of no channeling between the internal and external flow. Thus the sealing method was reasonable for the gap opening, and the sealing effect was well, which will ensure the internal and external flow not channeling.

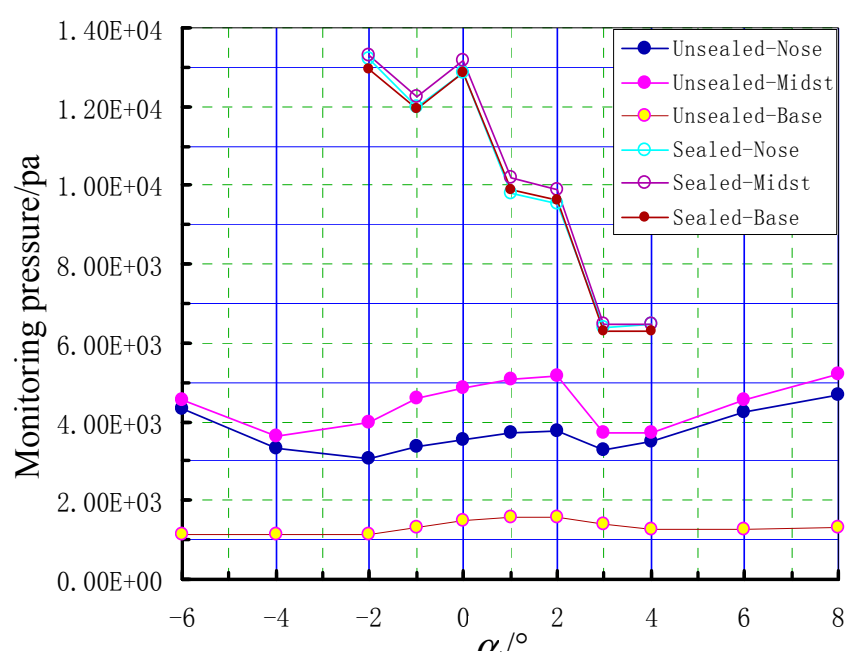

Figure 7. Monitoring pressure of clearance sealed vs unsealed

\subsection{Comparison of Test Results with or without the Internal and External Channeling}

The axial force coefficient curves $\left(C_{A}\right)$ for the tests with and without gap opening seal were shown in Figure 8 . 


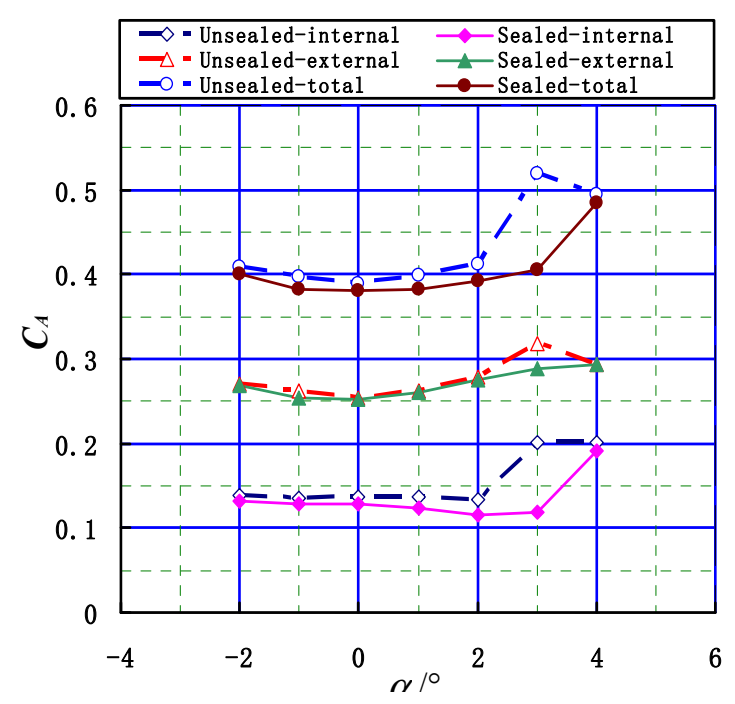

Figure 8. Axial-force coefficient of base clearance sealed vs unsealed

As shown in Figure 8, there was not the effect of channeling for the test with sealing. Within $\alpha=-2^{\circ} \sim 2^{\circ}$ angle of attack range during the inlet start, because of the consistent trend among the axial force coefficients of internal flow, external flow and whole model: the axial force coefficients increased with increasing the absolute value of angle of attack and were symmetrical around the ordinate. The axial force coefficient of the internal flow trends unusual for the test without sealing: the axial force coefficients increased with increasing the absolute value of angle of attack, which indicated that channeling influenced the internal flow aerodynamic.

The axial force coefficients for the gap opening with and without sealing trend well, indicating that the force generated by channeling was part of the internal forces, which has effect on the internal and external aerodynamics but has little effect on the entire missile aerodynamics.

\subsection{Comparison of Test Results for the Internal and External Flow Components with and Without Contact}

The axial force coefficient curves of the typical experiment tests for the internal and external flow decoupling and no decoupling (internal and external flow components were locally contacted) were shown in Figure 9. "Touch" denoted the tests for the internal and external flow components locally contacted, and "Normal" denoted the tests for the internal and external flow decoupling.

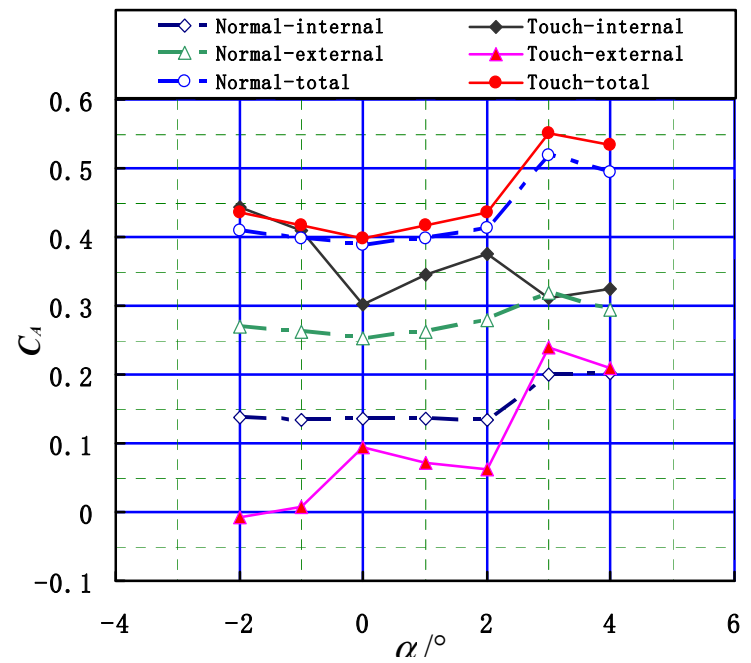

Figure 9. Axial-force coefficient of decoupled vs un-decoupled

It can be seen from Figure 9, there was not any law in the internal and external axial force coefficient curve 
changed with the angle of attack for the "Touch" tests with totally opposite trend and repeated sharp jump, indicating that the force was transmitted when the internal and external components were locally contacted. The axial force coefficient curves of the internal, external and the whole model trended consistent, which was consistent with the law, and proved that there was not channeling phenomenon and force transmission between the internal and external components, to achieve internal and external flow decoupled physically.

From Figure 9, the axial force coefficient curves of the entire model for the "Touch" and "Normal" tests coincided, indicating that the force transmission generated with the internal and external flow components contacts was part of internal forces, which had a great impact on the internal and external aerodynamic but no impact on the entire model. The axial flow coefficient of the internal flow for the "Touch" tests was much smaller than that for the "Normal" tests. The axial flow coefficient of the external flow for the "Touch" tests was much bigger than that for the "Normal" tests, which indicating that the internal and external components locally contacted, in the axial direction, the internal forces were delivered from the internal parts to the external components.

Comparing Figure 8 and Figure 9, it showed that, as compared with the internal forces generated by channeling, the axial force coefficient curves for the internal and external flow components locally contacted jumped acutely, which indicated the internal forces by channeling was far less than that of the internal and external flow components locally contacted.

The local contact for the internal and external flow components was due to the circuitry of pressure monitors. After the impact on the model by the circuitry of pressure monitors was excluded, there were not the tests in which the internal and external flow components locally contacted. The tests for the gap opening without sealing were for comparison analysis deliberated. During the test, the high temperature sealant did not come off. After the test finished, because the model was acutely vibrated, the high temperature exhausts gas oscillated back and forth, which led to $80 \%$ of the sealant off. Therefore, in order to monitor the sealing effect, the pressure monitoring points should be set in the cavity gap. The impact on the internal and external flow components by the circuitry of pressure monitors should be regarded, otherwise it would lead to the internal and external locally contact and force transmission.

\section{Conclusions}

In this paper, the test system design technology for the internal and external flow decoupling of the air-breathing hypersonic had been studied, and the validation tests were conducted under Mach 6 conditions, the following conclusions were achieved:

(a) The test had not channeling, the balance measurement data of the internal and external flow aerodynamic load were obtained for the condition without channeling and components contact, which prove the test system design was successful, solve the internal and external flow decoupling problems, and achieve internal and external flow aerodynamic decoupled physically.

(b)When the internal and external flow components were designed separately, the best location of the head gap opening passage was near the lip as close as possible; the opening size should be suitable to have little impact on the internal flow field structure, which cannot be sealed.

(c) In the conventional hypersonic wind tunnel, the high temperature sealant was used to seal the gap opening of the internal and external flow components, which can ensure the scale model not channeling and not passing force to achieve the internal and external flow decoupling.

(d) When there were force transmission and channeling for the internal and external flow components, the internal forces will be produced, which had impact on the internal and external flow aerodynamic but had no impact on the entire missile aerodynamics. The internal forces due to the internal and external flow contacted locally had greater effect on the internal and external flow aerodynamic as compared with that generated by channeling.

The future research is to develop the design technique for the aerodynamic model with rectangular cross section similar to that of X-51A. After the mature of the internal and external flow decoupling technology, the internal flow direct dynamometer test for the scramjet were conducted in the conventional hypersonic wind tunnel, the internal and external flow aerodynamics were measured simultaneously for the air-breathing hypersonic vehicle, and it was also applied in the tests that the segmented dynamometer and the thrust-resistance measurement tests were carried out on a scramjet test platform. 


\section{References}

Anderson, E. A. (1983, February). Mach 7 performance of the Langley airframe-integrated modular scramjet. JANNAF Propulsion Meeting, vol. V, CPIA Pub (370, pp. 493-508).

Bai, H. C., \& Wang, Z. J. (2012). Discussion on force-accounting system for air-breathing hypersonic vehicle. Journal of Propulsion Technology, 33(1), 1-6.

Chen, Y. H., \& Xia, H. (2004). Fidelity of the thrust performance in RJTF scramjet test. Aerodynamic Missile Journal, 10, 48-56.

Engelund, W. C., Holland, S. D., \& Charles E. (2000). Aerodynamic database development for the hyper-X airframe integrated scramjet propulsion experiments. AIAA-2000-4006. http://dx.doi.org/10.2514/6.2000-4006

Holland, S. D., Woods, W. C., \& Engelund, W. C. (2001). Hyper-X research vehicle experimental aerodynamics test program overview. Journal of Spacecraft and Rockets, 38(6), 11-12. http://dx.doi.org/10.2514/2.3772

Le, J. L. (2010). Progress in air-breathing hypersonic technology. Journal of Propulsion Technology, 31(6), 641-649.

Luo, J. L., Xu, M., \& Liu J. (2007). Integrated hypersonic vehicle shape up resistance characteristics. Journal of Astronautics, 28(6), 1478-1481.

Luo, S. B., Huang, W., Jing, L., \& Wang, Z. G. (2010, April). Drag force characteristic of a typical dual-mode scramjet combustor. International Conference on Computer Engineering and Technology- ICCET (V5, 255-259). Chendu, China. DOI: 10.1109/ICCET.2010.5485998

Mitani, T., Kobayashi, K., \& Hiraiwa T. (2001). Evaluation of internal aerodynamic performance in scramjet engines. AIAA-2001-1885.

Pinckney, S. Z. (1978). Internal performance predictions for langley scramjet engine module. NASA TMX-74038.

Tang, Z. G. (2004). Hypersonic aerodynamic test. Beijing, China: National Defense Industry Press.

Voland, R. T. (1990). Methods for determining the internal thrust of scramjet engine modules from experimental data. AIAA-90-2340.

Wang, F. X. (2001). High-speed wind tunnel test. Beijing, China: National Defense Industry Press.

Wang, Y. S. (2004). Three-component force measurement for scramjet engine (a) -drag measurement at $\mathrm{Ma}=4 \sim 8$ flight conditions. Aerodynamic Missile Journal, 7, 42-49.

Xu, X. B., Shu, H. F., Xie F., \& Zhang D. W. (2013). Technique investigation on flow through model inner flow drag straightway measured by strain-gauge balance. Journal of Propulsion Technology, 34(2), 311-315.

Yun, Q. L. (1991). Experimental aerodynamics. Beijing, China: National Defense Industry Press.

\section{Copyrights}

Copyright for this article is retained by the author(s), with first publication rights granted to the journal.

This is an open-access article distributed under the terms and conditions of the Creative Commons Attribution license (http://creativecommons.org/licenses/by/3.0/). 